\title{
COBERTURA REAL DE LA LEY DE ATENCIÓN DE EMERGENCIA Y DEL SEGURO OBLIGATORIO CONTRA ACCIDENTES DE TRÁNSITO (SOAT)
}

\author{
J. Jaime Miranda ${ }^{1,2,3,4, a}$, Edmundo Rosales-Mayor ${ }^{1,2,5,6, b}$, Camila Gianella ${ }^{1,4, c}$, Ada Paca-Palao ${ }^{1, d}$, \\ Diego Luna ${ }^{1,7,8, e}$, Luis Lopez ${ }^{1,9, f}$, Luis Huicho ${ }^{1,10,11,9}$, Equipo PIAT ${ }^{\S}$
}

\begin{abstract}
RESUMEN
Objetivo. Determinar, desde la perspectiva de los pacientes, el grado de conocimiento y de cobertura real de la Ley de Atención de Emergencia y del Seguro Obligatorio Contra Accidentes de Tránsito (SOAT). Materiales y métodos. Estudio transversal de vigilancia activa en los servicios de emergencia de establecimientos de salud (EESS) de tres ciudades del país con heterogeneidad económica, social y cultural (Lima, Pucallpa y Ayacucho). Resultados. De 644 encuestados, $77 \%$ negaron conocer la Ley de Atención de Emergencia (81\% en Lima, 64\% en Pucallpa y $93 \%$ en Ayacucho; $p<0,001$ ). Luego de explicarles dicha ley, $46 \%$ del total respondió que fueron atendidos bajo los preceptos de la ley. Con respecto al SOAT; en 237 personas $(37,2 \%)$ los gastos de atención no fueron cubiertos por un seguro ( $74 \%$ en Pucallpa, $34 \%$ en Ayacucho y $26 \%$ en Lima: $p<0,001$ ). De los que contaban con un seguro (SOAT u otro), pero cuya atención no fue cubierta por éste, la mayoría estuvo en Lima (70\%), seguido de Ayacucho (30\%) y ninguno en Pucallpa. La mayoría (94\%) procedió de establecimientos públicos. Las razones incluyeron problemas administrativos $(25 \%)$, vencimiento del seguro o morosidad en el pago (21\%), invalidez del seguro en el establecimiento (18\%), no disponibilidad de la póliza (18\%), o no cobertura de las lesiones por el seguro (9\%). En estos casos, los gastos fueron asumidos por el mismo paciente, familiares o parientes, el chofer o dueño del vehículo, el servicio social del EESS, u otras personas o instituciones. Conclusiones. En este estudio se observó un gran desconocimiento de la Ley de Atención de Emergencia, y una cobertura de atención muy deficiente, pues casi la mitad de atenciones no fueron brindadas de acuerdo a uno o más puntos establecidos por dicha norma. Los costos de atención generados por el accidente de tránsito no fueron cubiertos por un seguro en una de cada tres víctimas. Es urgente mejorar la información de los ciudadanos sobre sus derechos y el cumplimiento efectivo de las leyes, para lograr una cobertura universal y más equitativa en la atención de las víctimas de accidentes de tránsito.
\end{abstract}

Palabras clave: Servicios médicos de urgencia; Aplicación de la ley; Seguro por accidentes; Beneficios del seguro; Accidentes de tránsito; Perú (fuente: DeCS BIREME).

\section{COVERAGE OF THE EMERGENCY HEALTH CARE LAW AND THE COMPULSORY INSURANCE AGAINST ROAD TRAFFIC CRASHES (SOAT)}

\begin{abstract}
Objective. The aim of this study was to ascertain, from patients' perspective, the degree of knowledge and the actual coverage of the Emergency Health Care Law and the Compulsory Insurance against Road Traffic Crashes (SOAT). Material and methods. A cross-sectional, active surveillance of emergency wards of selected health facilities in three Peruvian cities (Lima, Pucallpa y Ayacucho) was conducted. Results. Out of 644 surveyed victims, $77 \%$ did not know about the law about provision of emergency health care (81\% in Lima, $64 \%$ in Pucallpa y $93 \%$ in Ayacucho; $p<0,001)$. Following the explanation of what this law entails, $46 \%$ reported to have received care according to the law specifications.
\end{abstract}

\footnotetext{
Programa de Investigación en Accidentes de Tránsito, Salud Sin Límites Perú. Lima, Perú.

Facultad de Medicina, Universidad Peruana Cayetano Heredia. Lima, Perú.

CRONICAS, Centro de Excelencia en Enfermedades Crónicas, Universidad Peruana Cayetano Heredia. Lima, Perú.

EDHUCASALUD, Asociación Civil para la Educación en Derechos Humanos con Aplicación en Salud. Lima, Perú.

Centro de Trastornos Respiratorios del Sueño (CENTRES), Clínica Anglo Americana. Lima, Perú.

Grupo de Investigación en Sueño (GIS). Lima, Perú.

Departamento de Ciencias Sociales y Políticas, Universidad del Pacífico. Lima, Perú.

Asociación Civil "Gobierno Coherente". Lima, Perú.

Dirección de Formación Profesional y los Recursos Humanos, Ministerio del Trabajo y Promoción del Empleo. Lima, Perú.

10 Departamento de Pediatría, Instituto Nacional de Salud del Niño. Lima, Perú.

11 Facultad de Medicina, "San Fernando" Universidad Nacional Mayor de San Marcos. Lima, Perú.

a Médico, Magíster y Doctor en Epidemiología; ${ }^{b}$ Médico, Magíster en Medicina y Magíster en Sueño: Fisiología y Medicina; ${ }^{\circ}$ Licenciada en

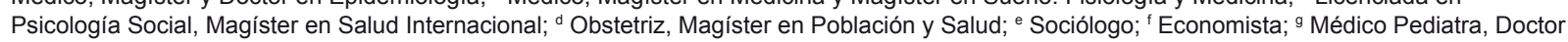
en Medicina.

$\S$ Ver sección agradecimientos para la lista completa de participantes del Equipo PIAT.
}

Recibido: 08-04-10 Aprobado: 02-06-10 
As for SOAT, the health care related costs of 237 persons $(37.2 \%)$ were not covered by any insurance scheme $(74 \%$ in Pucallpa, $34 \%$ in Ayacucho and $26 \%$ in Lima: $p<0,001)$. Conclusions. In this study, the lack of knowledge about the provision of emergency health care law was important, and the coverage of care was deficient as nearly half of participants reported not to be treated by one or more of the entitlements stated in such law. Road traffic injuriesrelated health care costs were not covered by any insurance scheme in one of three victims. Improvements on citizens' information about their rights and of effective law enforcement are badly needed to reach a universal and more equitable coverage in the health care of road traffic-related victims.

Key words: Emergency medical services; Law enforcement; Accident insurance; Insurance benefits; Accidents, traffic; Peru (source: MeSH NLM).

\section{INTRODUCCIÓN}

En el Perú, las estadísticas disponibles con relación a los accidentes de tránsito no son nada alentadoras: en el año 2008 ocurrieron 3489 muertes y 49440 heridos debido a accidentes de tránsito a nivel nacional (1). La mayoría de las víctimas son principalmente varones ( $71 \%$ ) y mayores de 18 años ( $79 \%)$, es decir, la población económicamente activa ${ }^{(1,2)}$

La atención de las emergencias en el Perú es por ley obligatoria y prioritaria, y no puede ser condicionada al pago previo en ningún establecimiento de salud público o privado ${ }^{(3)}$. Esto se encuentra contemplado en la Ley General de Salud ${ }^{(4,5)}$, comúnmente Ilamada "Ley de Atención de Emergencia".

Por otra parte, el Seguro Obligatorio de Accidentes de Tránsito (SOAT) se puso en vigencia desde el 1 de junio del año 2002, con la finalidad previsional de permitir la atención de las víctimas de accidentes de tránsito, tanto para los ocupantes del vehículo o vehículos involucrados, pero también para los peatones que pudieran verse afectados. Este seguro tiene como objetivo garantizar la atención, de manera inmediata e incondicional, de las víctimas de accidentes de tránsito que sufren lesiones corporales y muerte ${ }^{(6,7)}$. Tanto la Ley de Atención de Emergencia como el SOAT, al ser obligatorios para los prestadores de salud y para todos los vehículos que circulan por las carreteras del país, respectivamente, deberían garantizar el acceso universal a la atención de salud, de todas las victimas de un accidente de tránsito. Sin embargo, existen diversas razones por las que su cumplimiento puede no ser efectivo, ya sean razones derivadas de los ciudadanos potencialmente beneficiarios, como razones provenientes de los obligados a cumplirlos.

El objetivo del presente estudio fue evaluar el conocimiento de la Ley de Atención de Emergencia y su cumplimiento $y$, por otro lado, evaluar si las atenciones fueron cubiertas por el SOAT en personas víctimas de un accidente de tránsito.

\section{MATERIALES Y MÉTODOS}

\section{TIPO DE ESTUDIO}

Estudio transversal, mediante vigilancia activa in-situ de todos los accidentes de tránsito en un periodo prospectivo de cuatro semanas, en los servicios de emergencia de establecimientos de salud (EESS) seleccionados en tres ciudades del país: Ayacucho, Lima y Pucallpa.

\section{POBLACIÓN}

En cada localidad fueron seleccionados los EESS, públicos y privados más importantes, tanto por su cercanía a vías de comunicación (carreteras o autopistas) de acceso a la ciudad como por tener una tasa promedio de atenciones de víctimas de accidentes de tránsito mayor a 2/día o mayor a 60/mes, de acuerdo con las estadísticas del EESS de uno o dos años previos al estudio. Las clínicas fueron consideradas como EESS privados y los hospitales como EESS públicos.

Participaron de la investigación aquellas personas que acudieron al EESS seleccionado por lesiones provocadas por o vinculadas a un accidente de tránsito durante el período de vigilancia de cuatro semanas consecutivas, entre agosto y septiembre de 2009.

Se usaron los siguientes criterios de inclusión: personas que hubieran sido atendidas en el EESS, personas mayores de edad (18 años o más), que el lesionado o un familiar pudieran comunicarse en castellano, y personas que no presentaran alteraciones del estado de conciencia ni del habla.

\section{RECOLECCIÓN DE DATOS}

Se utilizó un cuestionario semiestructurado dirigido para evaluar tanto el conocimiento como el cumplimiento de la Ley de Atención de Emergencia. Los encuestadores fueron capacitados en el uso de los instrumentos y cubrieron dos turnos ( 7.00 a 13.00 horas y 13.00 a 19.00 horas) de lunes a sábado para su aplicación. Se planificó una estrategia de contacto y visitas domiciliarias para ubicar a aquellos 
pacientes que fueron admitidos o dados de alta en los horarios o días no cubiertos por los encuestadores. El objetivo fue tener una cobertura del $100 \%$ de las víctimas de accidentes de tránsito que acudieran a los EESS seleccionados en el tiempo de estudio.

Para evaluar el cumplimiento de esta Ley se plantearon cuatro situaciones contempladas en ella:

- Situación 1. Los EESS están obligados a prestar atención inmediata;

- Situación 2. La determinación de emergencia es realizada por profesional médico;

- Situación 3. Cuando el EESS no pueda brindar los recursos necesarios para la atención especializada, se procederá a llamar a un profesional especialista o se transferirá al paciente;

- Situación 4. El pago por concepto de atención de emergencia, se realizará luego de la atención.

Para el análisis, se consideró que no se había cumplido con atender bajo los preceptos de la Ley si el participante había respondido de manera negativa a la pregunta sobre si había sido atendido según la Ley en una o más de las cuatro situaciones planteadas. Además, se hizo un análisis por cada una de las situaciones planteadas. Ambos análisis se hicieron en toda la población y por cada ciudad.

Con relación al SOAT, se hicieron preguntas evaluando si la atención recibida por el encuestado fue efectivamente cubierta por este seguro. De no haber sido el caso, se le pidió a cada participante que explicara los motivos.

\section{CONSIDERACIONES ÉTICAS}

El protocolo fue revisado y aprobado por el Comité de Ética en Investigación del Instituto Nacional de Salud, y además por los comités de ética en investigación en los EESS que contaban con ellos. El objetivo del estudio fue explicado a cada uno de los participantes y se obtuvo el consentimiento informado escrito previo a la aplicación del cuestionario.

\section{ANÁLISIS DE DATOS}

Los datos recolectados fueron ingresados a una tabla del programa Microsoft Excel v.11 (Microsoft Corp, Redmond, Washington, EEUU), y el análisis estadístico fue realizado en el programa Stata 10.0 (STATA Corp, College Station, Texas, EEUU). La comparación de proporciones se analizó utilizando la prueba de Ji Cuadrado o el test exacto de Fisher, según sea conveniente. Se consideró significancia estadística $p<0,05$.

\section{RESULTADOS}

Los EESS públicos que participaron fueron: Hospital Regional de Ayacucho (Ayacucho), Hospital de Apoyo de Yarinacocha (Pucallpa), Hospital Regional de Pucallpa (Pucallpa), Hospital Nacional Hipolito Unanue (Lima), Hospital Nacional Dos de Mayo (Lima), y Hospital Nacional Cayetano Heredia (Lima). Y los EESS privados fueron: Clinica Montefiori (Lima) y Clinica Maison de Santé (Lima). Fueron encuestadas 644 personas (Tabla 1 ), la tasa de respuesta global fue de $82 \%$ (Figura 1). El $46 \%$ de los encuestados eran mujeres y la edad promedio fue $37,3 \pm 15,7$ años (rango 18 - 88 años). De las 138 personas elegibles que no fueron encuestadas, $40 \%$ eran mujeres y la edad promedio fue $39,4 \pm$ 15,7 años (rango $18-87$ años). No hubo diferencias significativas en cuanto a edad y sexo en las personas no encuestadas.

\section{CONOCIMIENTO DE LA LEY DE ATENCIÓN DE EMERGENCIAS}

De los 644 encuestados, $77 \%$ negó conocer la Ley de Atención de Emergencia, 21\% afirmó conocerla y el resto $(2 \%)$ no respondió. Negó conocer la Ley en Lima el $81 \%$ de los encuestados, en Pucallpa el $64 \%$ y en Ayacucho el 93\% ( $p<0,001)$.

Tabla 1. Distribución de personas encuestadas según ciudad y tipo de establecimiento de salud (EESS).

\begin{tabular}{|c|c|c|c|c|}
\hline & $\begin{array}{c}\text { Lima } \\
\mathrm{N}=444(69 \%)\end{array}$ & $\begin{array}{c}\text { Pucallpa } \\
\mathrm{N}=141(9 \%)\end{array}$ & $\begin{array}{c}\text { Ayacucho } \\
N=59(22 \%)\end{array}$ & $\begin{array}{c}\text { Total } \\
\mathrm{N}=644(100 \%)\end{array}$ \\
\hline EESS Público & & & & $\mathrm{N}=522(81 \%)$ \\
\hline Hospital Nacional Cayetano Heredia & $N=103$ & -- & -- & -- \\
\hline Hospital Nacional Dos de Mayo & $N=86$ & -- & -- & -- \\
\hline Hospital Nacional Hipólito Unanue & $N=133$ & -- & -- & -- \\
\hline Hospital Regional de Pucallpa & -- & $\mathrm{N}=89$ & -- & -- \\
\hline Hospital Amazónico de Yarinacocha & -- & $\mathrm{N}=52$ & -- & -- \\
\hline Hospital Regional de Ayacucho & -- & -- & $N=59$ & -- \\
\hline EESS Privado & & & & $\mathrm{N}=122(19 \%)$ \\
\hline Clínica Montefiori & $N=81$ & -- & -- & -- \\
\hline Clínica Maisón de Santé Este & $\mathrm{N}=41$ & -- & -- & -- \\
\hline
\end{tabular}




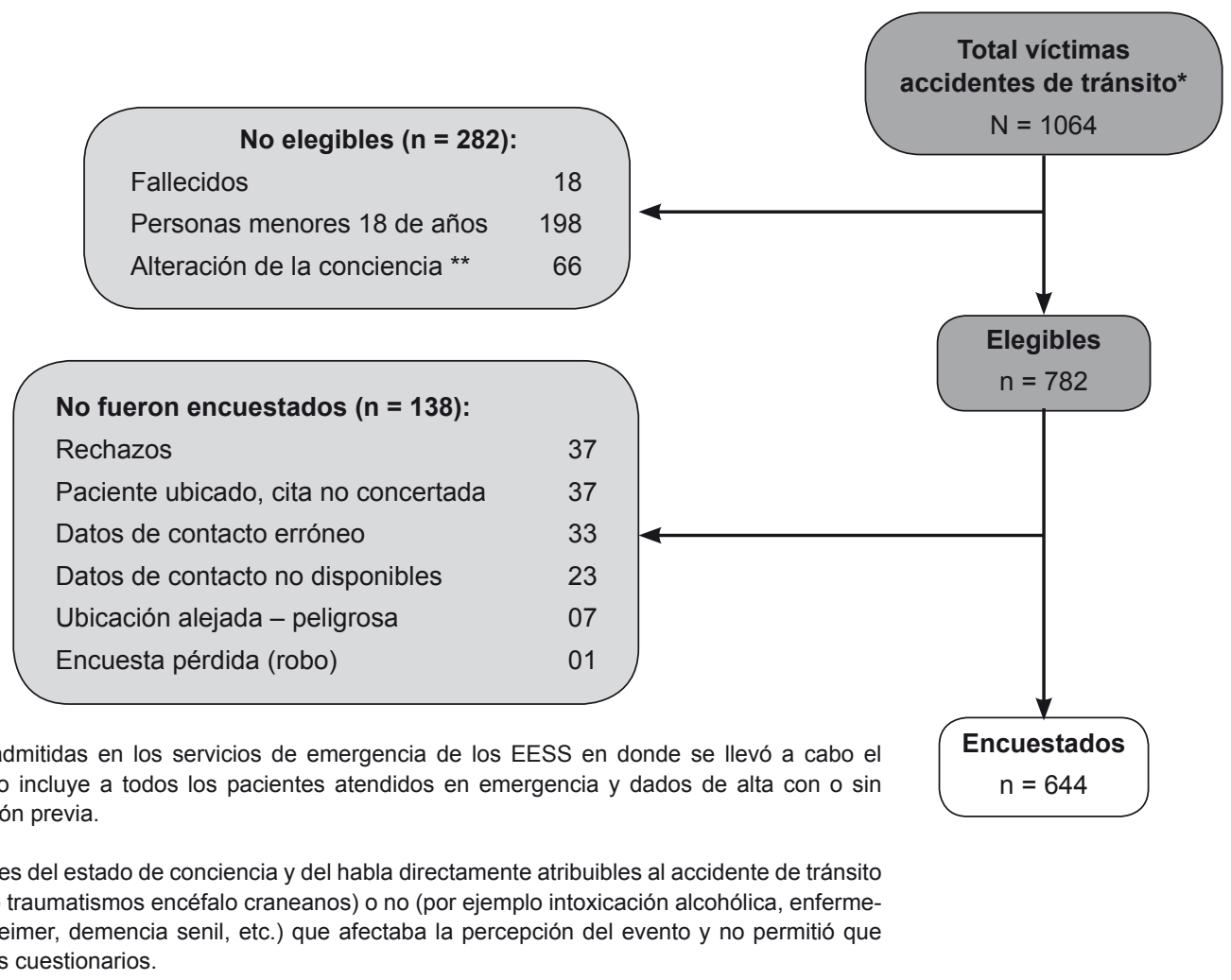

* Víctimas admitidas en los servicios de emergencia de los EESS en donde se llevó a cabo el estudio. Esto incluye a todos los pacientes atendidos en emergencia y dados de alta con o sin hospitalización previa.

** Alteraciones del estado de conciencia y del habla directamente atribuibles al accidente de tránsito (por ejemplo traumatismos encéfalo craneanos) o no (por ejemplo intoxicación alcohólica, enfermedad de Alzheimer, demencia senil, etc.) que afectaba la percepción del evento y no permitió que contesten los cuestionarios.

Figura 1. Flujograma de participantes en el estudio

\section{CUMPLIMIENTO DE LA LEY DE ATENCIÓN DE EMERGENCIAS}

De los 644 encuestados, 294 (46\%) respondieron que sí fueron atendidos bajo dichos preceptos de la ley, 285 (44\%) respondieron de manera negativa y 65 (10\%) no respondieron a la pregunta: 7 de Lima, 6 de Pucallpa y 52 de Ayacucho. Sin considerar a las personas que no respondieron a la pregunta, se muestra en la Tabla 2 la proporción de respuestas negativas a la pregunta si fueron atendidos bajo los preceptos de la Ley para cada una de las cuatro situaciones planteadas.

\section{SOAT Y COBERTURA DE GASTOS DE ATENCIÓN}

Todos los encuestados fueron consultados sobre si los gastos de la atención recibida en el EESS habían sido cubiertos por un seguro médico (Figura 2).

Tabla 2. Proporción de respuestas negativas a la pregunta de si fueron atendidos bajo los preceptos de la Ley de Atención de Emergencias*

\begin{tabular}{|c|c|c|c|c|c|}
\hline Ley de Atención de Emergencia & $\begin{array}{c}\text { Lima } \\
\mathrm{N}=437\end{array}$ & $\begin{array}{c}\text { Pucallpa } \\
\mathrm{N}=135\end{array}$ & $\begin{array}{c}\text { Ayacucho } \\
\mathrm{N}=7\end{array}$ & $\mathbf{p}$ п & $\begin{array}{c}\text { Total } \\
\mathrm{N}=579\end{array}$ \\
\hline Situación 1: los EESS están obligados a prestar atención inmediata & $21 \%$ & $7 \%$ & $29 \%$ & $<0,001$ & $18 \%$ \\
\hline $\begin{array}{l}\text { Situación 2: la determinación de emergencia es realizada por profesional } \\
\text { médico }\end{array}$ & $11 \%$ & $3 \%$ & $0 \%$ & 0,011 & $9 \%$ \\
\hline $\begin{array}{l}\text { Situación 3: cuando el EESS no pueda brindar los recursos necesarios para la } \\
\text { atención especializada, se procederá a llamar a un profesional especialista o se } \\
\text { transferirá al paciente }\end{array}$ & $9 \%$ & $10 \%$ & $0 \%$ & 0,871 & $10 \%$ \\
\hline $\begin{array}{l}\text { Situación 4: el pago por concepto de atención de emergencia, se realizará luego } \\
\text { de la atención }\end{array}$ & $39 \%$ & $39 \%$ & $14 \%$ & 0,494 & $39 \%$ \\
\hline Todas las situaciones $†$ & $49 \%$ & $51 \%$ & $29 \%$ & 0,577 & $49 \%$ \\
\hline
\end{tabular}

* No se incluyó en el análisis a las 65 personas que no respondieron a la pregunta. ๆ Test exacto de Fisher. † Si el encuestado respondió de forma negativa en una o más de las cuatro situaciones. 


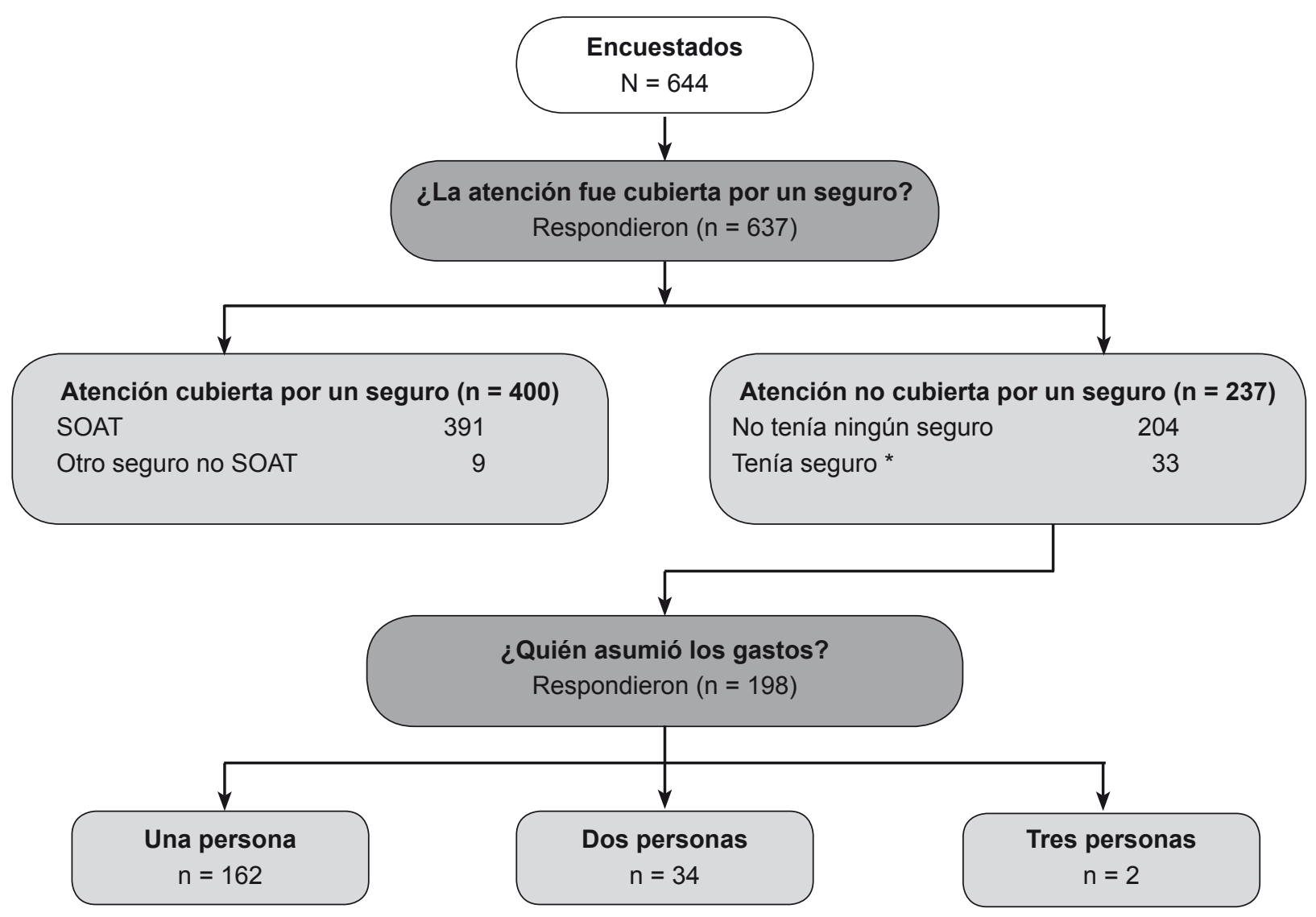

*Los encuestados refirieron que sí tenían un seguro, pero que la atención no fue cubierta por dicho seguro.

Figura 2. Proporción de atenciones cubiertas por un seguro y perfiles de gastos de atención en los casos sin seguro o sin cobertura efectiva.

Las 237 personas (37\%) cuyos gastos de atención por un accidente de tránsito no fueron cubiertos por un seguro se distribuyeron de la siguiente manera: $74 \%$ en Pucallpa, $34 \%$ en Ayacucho y $26 \%$ en Lima $(p<0,001)$.

Un grupo de personas refirió que contaba con un seguro (SOAT u otro seguro contra accidentes) al momento de la ocurrencia del accidente de tránsito pero su atención no fue cubierta por dicho seguro (Figura 2). La mayor parte de este grupo se encontraba en Lima (23 casos, $70 \%$ ), seguido de Ayacucho (diez casos, 30\%) y ninguno en Pucallpa. La gran mayoría (31 casos, 94\%) procedió de establecimientos públicos. Mediante una pregunta de opción múltiple, se preguntó a esas 33 personas sobre el motivo de dicha discrepancia, ocho (25\%) manifestaron que problemas administrativos no pudieron atenderlo con el seguro, siete $(21 \%)$ que el seguro se había vencido o no lo había pagado, seis $(18 \%)$ que el seguro no era válido en ese establecimiento, seis (18\%) que tenían seguro pero no presentaron la póliza correspondiente, y tres $(9 \%)$ que su seguro no cubría las lesiones que presentaban. Tres personas $(9 \%)$ no respondieron a la pregunta.

\section{PAGO DE LA ATENCIÓN}

En el grupo de personas cuya atención no fue cubierta por un seguro (Figura 2), una sola persona asumió los gastos en 162 casos, siendo su perfil variado: en $38 \%$ fue el mismo paciente, en $34 \%$ fueron los familiares o parientes del paciente, en $22 \%$ fue el chofer o dueño del vehículo causante del accidente de tránsito, en 3,5\% fue el servicio social del EESS donde se atendió, y en los cuatro casos restantes $(2,5 \%)$ fueron otras personas o instituciones (en tres casos fue la empresa en la cual trabajaba el paciente y en un solo caso el pago lo hizo el propio personal del EESS). De los casos en los que dos personas cubrieron los gastos (34 casos), en $74 \%$ fueron el paciente con sus familiares los que hicieron los pagos por la atención. En el resto de los casos restantes $(26 \%)$, fueron el chofer o dueño junto con la víctima o el familiar los encargados de hacer los pagos respectivos. Finalmente, de los dos casos en donde más 
de dos personas cubrieron los gastos de la atención, fueron principalmente el paciente con sus familiares los que hicieron los gastos, junto con el chofer o dueño del vehículo involucrado en el accidentes de tránsito o el servicio social del hospital.

\section{DISCUSIÓN}

Este estudio muestra una cobertura limitada de la Ley de Atención de Emergencia, ya sea por desconocimiento de los potenciales beneficiarios, como de diversos obstáculos para su cumplimiento efectivo. Un mayor grado de conocimiento de la ley por los ciudadanos y sobre todo una aplicación efectiva, podría reducir significativamente los gastos de bolsillo que los afectados realizan.

De igual modo, es preocupante que a pesar de la obligatoriedad de cobertura de seguro contra los accidentes de tránsito establecida por el SOAT, más de la tercera parte (36\%) de los participantes haya tenido que cubrir directamente los gastos de atención generados por el accidente de tránsito al momento de la atención de emergencia. Un estudio mostró que, en términos económicos, el principal financiador del gasto generado por la atención de emergencia y la hospitalización, en los casos en que fue necesario, era el SOAT ${ }^{(8)}$. El promedio del monto cubierto por el SOAT Ilegaba a unos 1046 nuevos soles. En esa misma investigación, también se pudo determinar que en las fases de convalecencia o rehabilitación son el paciente y su familia los que asumen de manera significativa esos costos, seguidos por el SOAT. Basado en lo anterior se puede ver el rol económico que asumirían el paciente y su familia cuando no se da la cobertura del SOAT.

Esta investigación brinda suficiente evidencia, aunque indirecta, de varias deficiencias que afectan la calidad de atención de las víctimas de accidentes de tránsito.

Primero, el beneficio esperado de este seguro obligatorio -en términos de lograr que la mayoría de la población víctima de un accidentes de tránsito tenga una cobertura de los gastos de atención iniciales generados por el accidente de tránsito- no alcanza al $100 \%$ de afectados. A fin de evitar inequidades se hace necesaria una revisión crítica del perfil de aquellos no beneficiados por una política pública de alcance, en teoría, universal.

Segundo, el estudio reporta una serie de deficiencias o barreras logísticas, operativas o administrativas que podrían ser resueltas de manera sencilla si se estableciesen mecanismos de diálogo y de información adecuados. Por ejemplo, en aquellos casos en los que el hospital "exige" la póliza, considerando que el "sticker" de tenencia del SOAT no es suficiente, demuestran una interpretación incorrecta de los alcances de este seguro. En estas circunstancias, el EESS debería atender de manera inmediata al afectado, que se encuentra en una condición de emergencia, tal como se indica en la Ley General de Salud ${ }^{(4,5)}$ y en la Norma Técnica de Salud de los Servicios de Emergencia ${ }^{(9)}$. El seguro sirve para cubrir económicamente la atención de la víctima, y el cobro por el servicio de salud, de acuerdo al artículo 11 de la Ley de Atención de Emergencia ${ }^{(5)}$ debe ser posterior a la atención.

Tercero, existe el Fondo de Compensación del SOAT ${ }^{(10)}$, el cual es administrado por un comité formado por el Ministerio de Transportes y Comunicaciones (MTC), quien lo preside, la Asociación Peruana de Empresas de Seguros (APESEG) y por la Superintendencia de Banca y Seguros (SBS). Este fondo fue creado con la finalidad de amparar a las víctimas de los accidentes de tránsito ocasionados por vehículos que no hayan sido identificados y se hayan dado a la fuga luego del evento, es decir, a aquellas personas a las que el SOAT no va a poder cubrir con los gastos de atención. Cabe resaltar que este fondo cubre los gastos mediante el reembolso de los gastos médicos o de sepelio. Por lo tanto, debido a la importante proporción de afectados que terminan sufragando los costos de la atención y a que aproximadamente uno de cada tres afectados son desempleados ${ }^{(8,11)}$, es necesaria un revisión del funcionamiento de este fondo de compensación, de modo que pueda cubrir efectivamente a las víctimas de un accidente de tránsito que cuentan con pocos recursos económicos desde el momento de la atención y no después de haber culminado ésta.

Este estudio, al establecer la factibilidad de una vigilancia activa de víctimas de accidentes de tránsito, contribuye entonces a establecer un tipo de mecanismo de evaluación periódica, cuya necesidad resulta evidente, dada la relevancia de los hallazgos. Al revelar al grupo de población desfavorecida, esta investigación no intenta establecer acusaciones directas, sino por el contrario, sentar las bases para adoptar procesos de monitoreo y evaluación que apunten hacia una mejoría continua en beneficio de todos los involucrados.

Los EESS estudiados estuvieron restringidos a algunos de los principales EESS en cada ciudad, específicamente a los que reciben un gran número de víctimas de accidentes de tránsito. El pequeño número de establecimientos seleccionados disminuye la posibilidad generalización de los resultados a otros EESS a nivel nacional.

El haber realizado la vigilancia en los EESS, muy probablemente conllevó a un subregistro de las víctimas 
con lesiones leves, quienes pueden no haber acudido a un EESS. No es posible estimar la magnitud de este potencial sesgo, dado que no se cuenta con un sistema de información sólido en accidentes de tránsito ${ }^{(12)}$.

Esta investigación tiene las limitaciones conocidas de los estudios con cuestionarios: apreciación subjetiva del encuestado, sesgo de recuerdo y problemas con la veracidad de la información brindada. Dichos factores se intentaron controlar realizando la encuesta apenas terminada la atención, e implementando una encuesta anónima y voluntaria. En cuanto a los gastos de la atención, ya que no se hizo un seguimiento de los encuestados, no se pudo conocer si el seguro cubrió o no la totalidad de los gastos generados por el accidente de tránsito entre aquellas personas que fueron atendidas con un seguro (SOAT u otro seguro). Además, no se pudo conocer cuántos de los encuestados pudieron hacer un uso posterior del Fondo de Compensación del SOAT. Nuestro estudio, con el fin de asegurar una alta tasa de respuesta, excluyó a personas que podrían tener limitaciones para contestar al cuestionario, lo que en parte podría limitar su validez externa. Sin embargo, este grupo representó únicamente el 23\% (66/282) del total de personas no consideradas elegibles.

En este estudio se muestra que existe un gran desconocimiento de la Ley de Atención de Emergencia, y una cobertura de atención muy deficiente, pues casi la mitad de atenciones no fueron brindadas de acuerdo a uno o más puntos establecidos por dicha norma. Además, la atención no fue cubierta por un seguro en una de cada tres víctimas, siendo esto particularmente preocupante en la ciudad de Pucallpa (tres de cada cuatro víctimas).

El número importante de personas que tuvieron que sufragar directamente los gastos de atención revela que se necesitan diversas estrategias para vigilar estas prácticas y establecer mejoras. Estas incluyen la provisión de una mayor información para resolver dudas administrativas y logísticas con el SOAT en beneficio de los afectados, dar información sobre el Fondo de Compensación del SOAT a las víctimas o familiares, así como la necesidad de mejorar y hacer más eficiente el trámite administrativo necesario dentro de los EESS, para que la víctima de un accidente de tránsito pueda ser beneficiado realmente por una atención cubierta por el SOAT u otro seguro contra accidentes.

Quedan pendientes por investigar diversos temas relacionados; por ejemplo, es necesario conocer en detalle la proporción de los gastos cubiertos por el SOAT que sirvieron para solucionar los problemas médicos de la víctima, o si el SOAT fue suficiente para asegurar su recuperación o rehabilitación. Además, es de suma importancia conocer cuantas de las personas que podrían ser beneficiarias del Fondo de Compensación del SOAT realmente hacen uso de él.

El presente estudio mediante una vigilancia activa, aporta con una herramienta práctica de evaluación que informa sobre el desenvolvimiento actual de la atención de los afectados por un accidente de tránsito. Esta herramienta puede constituirse en una estrategia de vigilancia y evaluación y permite establecer pautas para el mejoramiento continuo a favor de las víctimas de accidentes de tránsito.

\section{AGRADECIMIENTOS}

Nuestro agradecimiento a todos aquellos involucrados con el PIAT, a los coordinadores y trabajadores de campo en cada una de las sedes y a todos los participantes del estudio.

Nuestro agradecimiento también a todas las personas que en distintas fases brindaron su apoyo: Eduardo Bedriñana (Salud Sin Límites Perú, Ayacucho), Lucie Ecker (Instituto de Investigación Nutricional, Lima), Fernando Llanos (Universidad Peruana Cayetano Heredia, Lima), Willy Lescano (U.S. Naval Medical Research Center Detachment, Lima), David Moore (Imperial College London, Londres), Pablo Perel (London School of Hygiene and Tropical Medicine, Londres), Jorge Rey de Castro (Universidad Peruana Cayetano Heredia, Lima), Ian Roberts (London School of Hygiene and Tropical Medicine, Londres), Paul Valdivia (Universidad Peruana Cayetano Heredia, Lima), Walter Valdivia (Ministerio de Economía y Finanzas, Lima).

\section{Miembros del Programa de Investigación en Accidentes de Tránsito (PIAT)}

Coordinadora PIAT: Ada Paca; Asistentes de Investigación: Luis López, Diego Luna, Edmundo Rosales; Investigador Asociado: Pablo Best; Otros miembros del Equipo PIAT: Pablo Best, Miriam Egúsquiza, Camila Gianella, Claudia Lema, Esperanza Ludeña, Investigadores Principales: J. Jaime Miranda, Luis Huicho.

\section{Fuente de Financiamiento}

Este estudio fue financiado por el Instituto Nacional Salud de Perú como parte del Programa de Investigación en Accidentes de Tránsito ejecutado por Salud Sin Límites Perú.

\section{Conflictos de Interés}

Los autores declaran no tener conflictos de interés en la publicación de este artículo. 


\section{REFERENCIAS BIBLIOGRÁFICAS}

1. Perú, Ministerio de Transportes y Comunicaciones. Perú: Número de víctimas de accidentes de tránsito fatales y no fatales por año, según características de las víctimas: 2000 - 2008. [Documento en Internet] Lima: MTC; 2009. [Fecha de acceso: 2 febrero 2010] Disponible en: http://www. mtc.gob.pe/estadisticas/ARCHIVOS/ESTADISTICA\%20 items/4-INDICADORES\%20Y\%20PUBLICACIONES/ OTROS/accidentes\%20-\%20transito/3.G.3.xls.

2. Huicho L, Trelles M, Gonzales F, Mendoza W, Miranda J. Mortality profiles in a country facing epidemiological transition: an analysis of registered data. BMC Public Health 2009;9:47.

3. Perú, Ministerio de Salud. MINSA recuerda que atención de emergencias y partos no puede condicionarse a pago previo. Lima 2006. [Página Web] Lima: MINSA; 2006. [Fecha de acceso: 2 febrero 2010] Disponible en: Disponible en: http://www.minsa.gob.pe/ocom/prensa/notadeprensa. asp?np_codigo $=3317 \&$ mes $=2 \&$ anio $=2006$.

4. Perú, Congreso de la República. Ley General de Salud. Ley N 26842 de 09 de julio de 1997. Lima: Congreso de la República; 1997.

5. Perú, Congreso de la República. Ley № 27604 que modifica la Ley General de Salud № 26842, respecto de la obligación de los establecimientos de salud a dar atención médica en caso de emergencias y partos.Ley $N^{\circ} 27604$ de 19 de diciembre 2001. Lima: Congreso de la República; 2001.

6. Perú, Ministerio de Transportes y Comunicaciones. Texto único ordenado del reglamento nacional de responsabilidad civil y seguros obligatorios por accidentes de tránsito. Decreto Supremo $N^{\circ}$ 024-2002-MTC de 13 de junio de 2002. Lima: MTC; 2002.
7. Seguro Obligatorio de Accidentes de Tránsito (SOAT). Todo sobre el SOAT. [Página web]. Lima, SOAT; 2007. [Fecha de acceso: 2 de fbrero 2010] Disponible en: http:// www.soat.com.pe/sobreelsoat.html.

8. Best P, Miranda JJ, Huicho L, Paca A, Luna D, Lopez L, et al. [Informe Técnico]. Lima: Instituto Nacional de Salud, Salud Sin Límites Perú; 2009.

9. Perú, Ministerio de Salud. Norma técnica de salud de los servicios de emergencia. NT N 042-MINSA / DGSP-V.01. Lima, MINSA; 2007.

10. Perú, Ministerio de Transportes y Comunicaciones. Reglamento del fondo de compensación del seguro obligatorio de accidentes de tránsito. Decreto Supremo $\mathrm{N}^{\circ}$ 024-2004-MTC de 11 de junio del 2004. Lima: MTC; 2004.

11. Best P, Miranda JJ, Huicho L, Paca A, Luna D, Lopez L, et al. [Informe Técnico]. Lima: Instituto Nacional de Salud, Salud Sin Límites Perú; 2009.

12. Miranda JJ, Huicho L, Paca A, Najarro L, Luna D, Lopez L, et al. Lima: Instituto Nacional de Salud, Salud Sin Límites Perú; 2009.

Correspondencia: J. Jaime Miranda, MD, MSc, PhD.

Dirección: Programa de Investigación en Accidentes de Tránsito (PIAT), Salud Sin Límites Perú, Calle Ugarte y Moscoso \# 450 Of. 601, Magdalena, Lima 17, Perú.

Teléfono: (511) 2615684

Correo electrónico: jaimemiranda@saludsinlimitesperu.org.pe; jaime.miranda@upch.pe

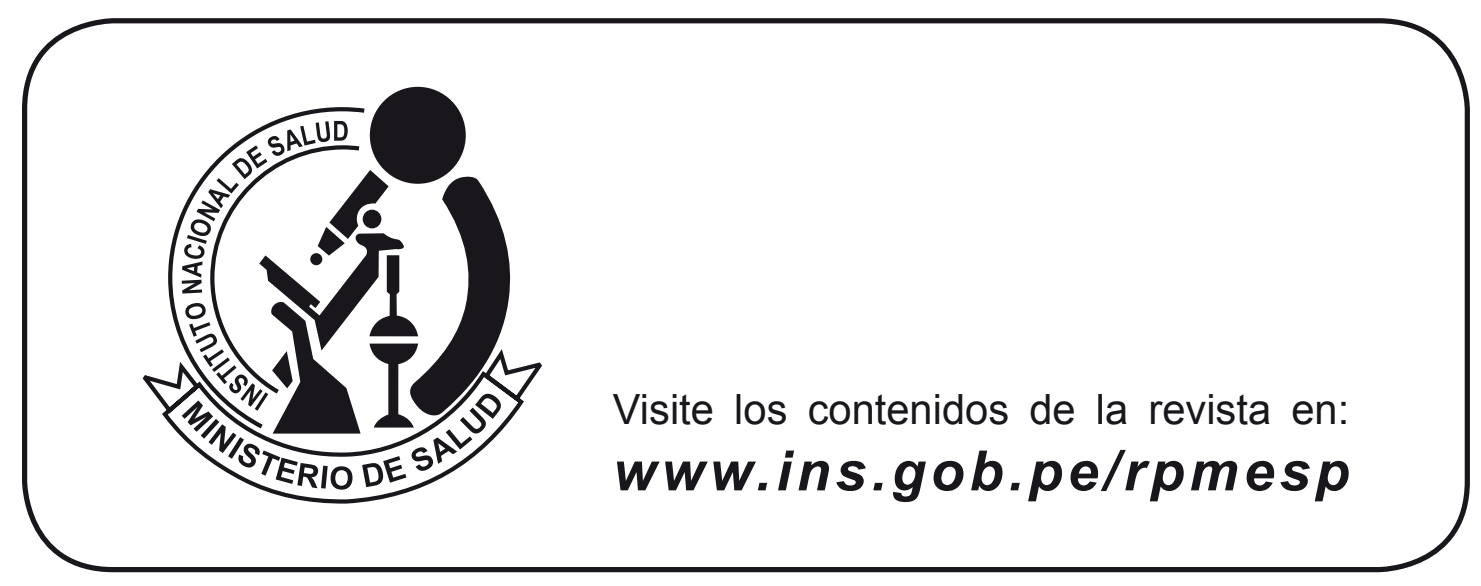

